\title{
Advanced Imaging Approaches to Reveal Molecular Mechanisms Governing Neuroendocrine Secretion
}

\author{
Lina Riachy $^{\mathrm{a}}$ Thomas Ferrand ${ }^{\mathrm{a}}$ Sylvette Chasserot-Golaz ${ }^{\mathrm{b}} \quad$ Ludovic Galas $^{\mathrm{c}}$ \\ Stéphane Alexandre ${ }^{d}$ Maité Montero-Hadjadje ${ }^{a}$ \\ aLaboratoire de Différenciation et Communication Neuronale et Neuroendocrine, Institut de Recherche et \\ d'Innovation Biomédicale de Normandie, Normandie University, UNIROUEN, INSERM, U1239, Rouen, France; \\ ${ }^{b}$ Institut des Neurosciences Cellulaires et Intégratives, Centre National de la Recherche Scientifique, Strasbourg \\ University, Strasbourg, France; 'Normandie University, UNIROUEN, INSERM, PRIMACEN, Rouen, France; \\ dPolymères, Biopolymères, Surfaces Laboratory, CNRS, Normandie University, UNIROUEN, UMR 6270, Rouen, France
}

\section{Keywords}

Super-resolution · Atomic force microscopy · Transmission electron microscopy · Total internal reflection fluorescence microscopy · Neuroendocrine cells $\cdot$ Regulated secretion · Neuroendocrinology $\cdot$ Cell biology

\begin{abstract}
Identification of the molecular mechanisms governing neuroendocrine secretion and resulting intercellular communication is one of the great challenges of cell biology to better understand organism physiology and neurosecretion disruption-related pathologies such as hypertension, neurodegenerative, or metabolic diseases. To visualize molecule distribution and dynamics at the nanoscale, many imaging approaches have been developed and are still emerging. In this review, we provide an overview of the pioneering studies using transmission electron microscopy, atomic force microscopy, total internal reflection microscopy, and superresolution microscopy in neuroendocrine cells to visualize molecular mechanisms driving neurosecretion processes, including exocytosis and associated fusion pores, endocytosis and associated recycling vesicles, and protein-protein or
\end{abstract}

protein-lipid interactions. Furthermore, the potential and the challenges of these different advanced imaging approaches for application in the study of neuroendocrine cell biology are discussed, aiming to guide researchers to select the best approach for their specific purpose around the crucial but not yet fully understood neurosecretion process.

(c) 2021 S. Karger AG, Basel

\section{Introduction}

Neuroendocrine secretion involves a succession of intracellular compartments, i.e., endoplasmic reticulum, Golgi apparatus and secretory granules, structures, and molecules [1], whose distribution and dynamics analysis require efficient resolutive imaging techniques. In neuroendocrine cells, secretory granules ensure the release of neurohormones/neuropeptides and neurotransmitters upon stimulation. These vesicular organelles, with a diameter ranging between 60 and $300 \mathrm{~nm}$ in their mature state depending on the neuroendocrine cell type [2], are generated by budding from the trans-Golgi membrane and convey their content to the cell periphery through a 
microtubule-/kinesin-dependent transport at a mean speed of $100 \mathrm{~nm} / \mathrm{s}$ [3]. Near the plasma membrane, actin filaments trap secretory granules through a myosin-dependent manner to keep a pool of vesicles ready for neuroendocrine cell response to extracellular solicitations [4]. Neuroendocrine cell stimulation provokes an increase in cytosolic calcium concentration and then the fast fusion of secretory granules with the plasma membrane, approximately $130 \mathrm{~ms}$, to release neurohormones more or less rapidly and totally (in a mean time of 1-2 s for a total release) into the blood stream [5]. Regulated exocytosis is a fast, multistep process controlled by calcium which, e.g., induces actin filament depolymerization and the activation of fusion proteins such as soluble N-ethylmaleimide-sensitive factor-attachment protein receptors (SNAREs) [6]. The major part of the molecular actors involved in exocytosis is sorted from the lumen and the membrane of the trans-Golgi compartment during secretory granule formation. At the level of this compartment in neuroendocrine cells, a family of proteins called granins, and among them more particularly chromogranin A ( CgA), plays a pivotal role by interacting with both (i) soluble neuropeptides, leading to their aggregation and sorting, and (ii) Golgi-membrane lipids and proteins, leading to their selection, to finally induce the Golgi-membrane budding. All of these events occur at the origin of functional secretory granule formation $[7$, 8].

The distinct steps of the secretory granule journey from biogenesis at the Golgi level to fusion at the plasma membrane need to be tracked with high spatial and temporal resolution to clarify the subtle molecular mechanisms underlying the vesicular/conventional neuroendocrine secretory pathway established in response to physiological needs [7]. The last two decades have seen a tremendous development in high-resolution microscopy techniques to visualize and track the molecular mechanisms governing the finely tuned steps of neuroendocrine secretion, e.g., fusion pore expansion. In this review, we will compile existing microscopy techniques, including total internal reflection fluorescence microscopy (TIRFM) and others which belong to the last developed superresolution microscopy (SRM) family, such as structured illumination microscopy (SIM) and stimulated emission depletion (STED), and which complement-confirmed techniques such as atomic force microscopy (AFM) and transmission electron microscopy (TEM). AFM is a powerful technique which specifically studies surfaces at the atomic or sub-nanometer scale [9]. TEM is used to spatially map plasma membrane domains through the prep- aration of plasma membrane sheets [10]. High-resolution imaging may also be achieved using SRM. This fluorescence technique was a major breakthrough for biological samples examination since, more recently, it has been used to overcome the optical diffraction barrier [11]. The impact of SRM was acknowledged when the scientists who developed the technique were awarded the Nobel Prize for Chemistry in 2014. The use of both AFM and SRM techniques is increasing in numerous fields including cell biology, plasmonics, catalysis, and biomaterials. The goal of all these techniques is to overcome the physical resolution barrier in order to determine precise molecule localization and, possibly, interactions at the level of cell membranes.

This review describes the technical advantages of using AFM, TEM, SRM, and TIRFM to study neuroendocrine secretion by highlighting the works that used these imaging approaches in neuroendocrine cells. Its main objective is to enable neuroendocrinologists to choose the most suitable microscopic method to analyze molecular mechanisms and interactions during the secretory process.

\section{Atomic Force Microscopy}

AFM is a member of a large family of surface-scanning probe microscopies introduced in 1980s by Binning et al. [12] with their invention of the scanning tunneling microscope. AFM has been applied in a wide range of disciplines, including solid-state physics [13], molecular engineering $[14,15]$, polymer chemistry and physics, surface chemistry, molecular biology and cell biology $[16,17]$, and medicine $[18,19]$. In biological domains, AFM is a powerful tool for visualizing and manipulating biological structures with nanometer resolution $[9,20]$. It can be used with light microscopes, especially in the biosciences [21]. An overview of the AFM method is shown in Figure 1. For example, AFM has been used on bacterial surfaces, revealing nanoscale net-like structures [22]. Since the direct "seeing" of single proteins and of eukaryotic-wallfree cell surface was a challenge for the first AFM, the ability to provide $3 \mathrm{D}$ information of the scanned surface led to its use on isolated protein complexes and assemblies, such as microtubules, to study their structure and mechanical properties [23]. AFM has been used on fixed whole cells (Fig. 1), e.g., to distinguish cancer cells and normal cells based on their hardness, to evaluate interactions between a specific cell and its neighboring cells in a competitive culture system [24], or to indent cancer cells in order to study how cells regulate the stiffness or shape 
Fig. 1. AFM and fusion pores. a Sharp tip fixed to the free end of the small spring-like cantilever is used to feel the sample surface. Optionally, a piezoelectric element (typically made of a ceramic material) oscillates the cantilever. The detector records the deflection and motion of the cantilever. The sample is mounted on the sample stage. A piezoelectric xyz scanner permits to displace the sample stage and the sample in $\mathrm{x}$, $y$, and $z$ directions with respect to the tip apex. Eventually, the $\mathrm{z}$ scanner may be used to oscillate the sample below the tip. Although this panel shows the drive attached to the sample, the drive can also be attached to the tip, or independent drives can be attached to both since it is the relative displacement of the sample and tip that needs to be controlled. Controllers and plotters are not shown here. The AFM signals, such as sample height, cantilever deflection, or phasing of the signal, are recorded on a computer during the $\mathrm{x}-\mathrm{y}$ scan. Eventually, during imaging, these informations are computed to obtain additional information on the physicochemical properties of the sample. These informations are plotted in a pseudocolor image (b), in which each pixel represents an $x-y$ position on the sample, and the color represents the recorded/ computed signal. AFM has three major abilities: imaging (topography, nanomechanics,...), force measurement, and manipulation. c Typical 3D representation of the cell surface topography. A portion of the cell surface contained in the delimited zone in (b) was scanned using AFM and revealed the presence of depressions with a dark-brown color on the plasma membrane of a chromaffin cell under acetylcholine stimulation. Some of these membrane deformations correspond to regulated exocytosis-related fusion pores with a width ranging between 90 and $130 \mathrm{~nm}$.

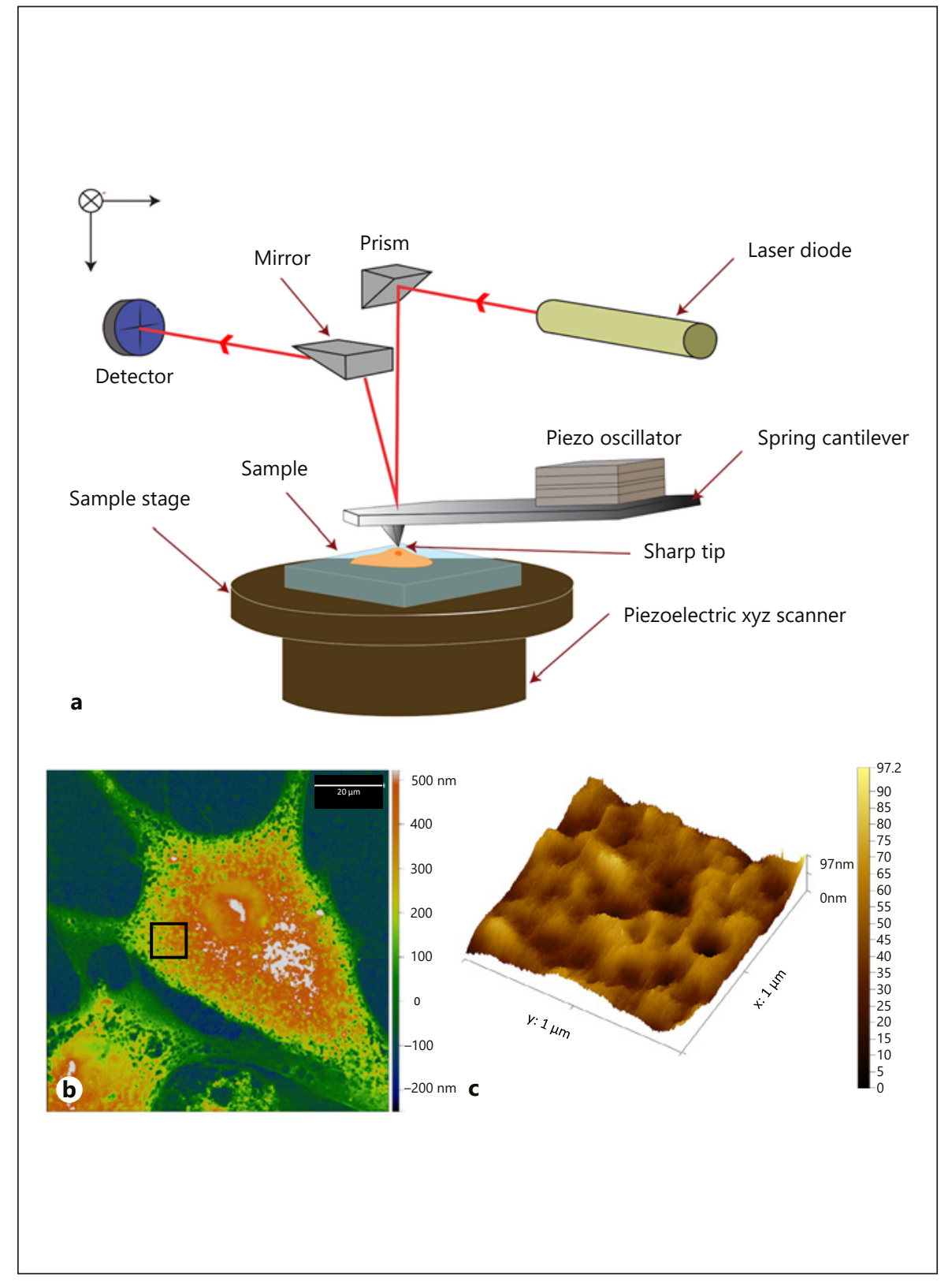

of their plasma membrane [25]. AFM can also be used to detect exocytotic fusion pores when neuroendocrine cells are stimulated [26] since, at the resting state, the cell surface is quite flat, with little fluctuations and, after secretion stimulation, the cell surface exhibits numerous depressions (Fig. 1). These plasma membrane depressions were identified using a primary antibody raised against a secretory granule transmembrane protein, recognized by a secondary antibody coupled to a $30-\mathrm{nm}$ gold particle. Exocytotic sites can be detected after cell stimulation, since the gold nanoparticles, observed at the level of plas- ma membrane depressions, reveal the number, membrane distribution, and morphological characteristics of fusion pores obtained using distinct types of secretagogues [26]. In another study, Tsai et al. [20] used AFM scanning to analyze endocytosis and vesicle recycling by measuring the size of the invagination pits. It must be noted that the scanning head may not reach the bottom of the invagination because of the limitation of the AFM tip length, leading to underestimation of the depth [25, 27]. AFM can also be used on plasma membrane artificial models. For example, a recent study demonstrated the 


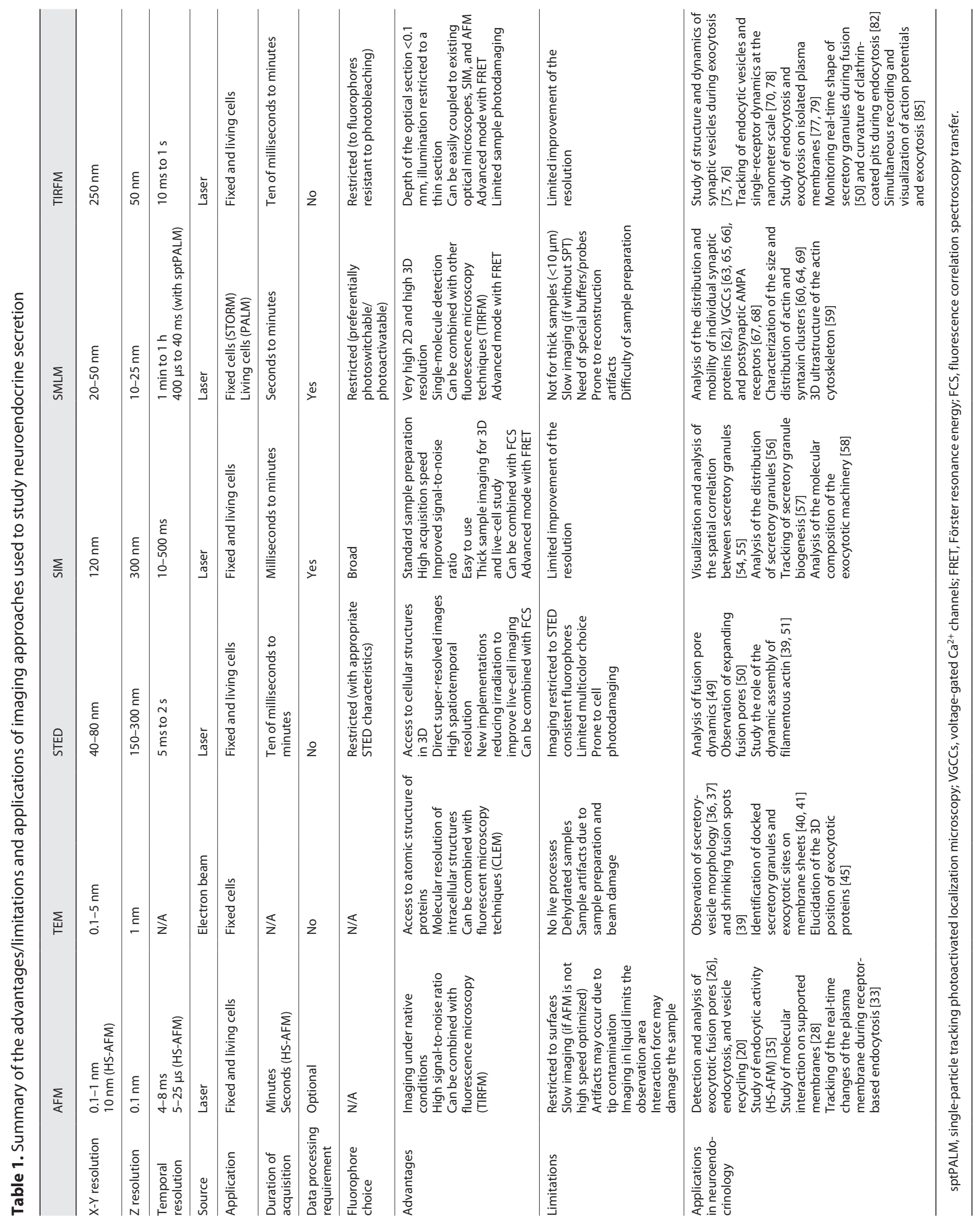


membrane molecular remodeling impact of a granin protein, CgA [28]. Using supported membrane bilayers, this study revealed that addition of the granular protein $\mathrm{CgA}$ to giant liposomes enriched with Golgi phosphatidic acid (PA) increased the number, height, and surface of thicker domains in a concentration and time-dependent manner [28]. This modification of membrane topography could result from either $\mathrm{CgA}$ aggregation, membrane deformation, microdomain formation, or any combination of these, indicating, in any case, that $\mathrm{CgA}$ induces the remodeling of the supported membrane bilayers through its interaction with Golgi PA.

Kelvin probe force microscopy mode is a derivative, noncontact mode of the AFM, allowing investigation of the electrical properties of materials, including biomolecules, using a conductive cantilever [29]. When the tip of the AFM is close to the plasma membrane, the surface potential caused by the charged molecules can be revealed at high resolution. In Tsai et al.'s [29] study, the AFM images revealed that the plasma membrane surface of neuroendocrine PC12 cells is not flat and exhibits a potential that is not homogenously distributed.

While AFM is more often applied to study the plasma membrane surface of fixed cells, its potentiality on living cells has been demonstrated [30, 31]. AFM using a PeakForce Tapping mode has been used to analyze structure, dynamics, and nanomechanical properties of the plasma membrane from living cells, which enables the visualization of microvilli of epithelial cells [32]. AFM using a nanomechanical measurement protocol enables researchers to follow real-time changes of the plasma membrane during receptor-based endocytosis in neuroendocrine cells [33]. More recently, high-speed AFM (HSAFM) has been developed to provide nanometer structural information and dynamics with subsecond resolution to analyze fast cellular events [34]. This AFM technique relies on the development of ultrashort cantilevers with microsecond time resolution and fast electronics and piezoelectric scanners which allow 1,000-fold faster imaging rates to be reached. As an example, HSAFM has been used to image living mammalian-cell endocytosis without damage [35].

All these studies demonstrate AFM's potential to dissect cell processes and molecular dynamics associated with exocytosis and endocytosis in neuroendocrine cells. AFM is a versatile tool which is in a state of constant evolution and improvement. In combination with other microscopy techniques (e.g., TIRFM), as detailed in the dedicated section below, it may provide further insights into the study of neuroendocrine secretion.

Innovative Imaging Techniques to Study

Neuroendocrine Secretion

\section{Transmission Electron Microscopy}

Although AFM is a powerful resolutive technique, only plasma membrane topography is achieved and is reconstituted after filtering during data analysis that might alter the original topographical features. TEM allows the earliest views of the plasma membrane through the use of electron-dense cellular stains and fixatives. In neuroendocrine cells, TEM has, for decades, focused on analysis of secretory-vesicle morphology [36]. The fusion model of the full collapse of the secretory-granule membrane with the plasma membrane during exocytosis was proposed on the basis of TEM data obtained 40 years ago [37]. This model was discarded very recently with the advent of super-resolution techniques allowing the study of living cells. Indeed, even if high-performance TEM still offers a resolution $\sim 10$ times higher (Table 1), current SRM techniques enabled to show shrinking fusion spots rather than growing fusion spots that subsequently disappear as would be expected from full collapse $[38,39]$.

TEM can also be used to study the molecular structure of membrane sheets prepared from resting or stimulated cells (Fig. 2) [10, 40]. This protocol enables determination of the number of secretory granules docked at or fused with the plasma membrane after cell stimulation. The use of immunogold labeling helps identify docked secretory granules and exocytotic sites on the inner face of the plasma membrane [41].

Using correlated light and electron microscopy (CLEM) by combining optical (fluorescence) microscopy and EM can improve the precise localization of molecules in subcellular compartments $[42,43]$. In an integrated CLEM system, the sample is imaged using an electron beam and a light path simultaneously. The electron microscope provides high-resolution information down to the nanoscale, while the fluorescence microscope highlights the regions of interest. This technique can be used to help find the 3D position of the endocytic protein epsin on clathrin-coated structures at the plasma membrane [44] or to uncover a relationship between the distance of secretory granules to the plasma membrane and the association between SNARE proteins of respective membranes [45].

\section{Super-Resolution Microscopies}

The advent of SRM allows observation of the plasma membrane deformations during exocytosis and endocytosis processes, observations vital to the study of molecu-

Neuroendocrinology 2023;113:107-119 


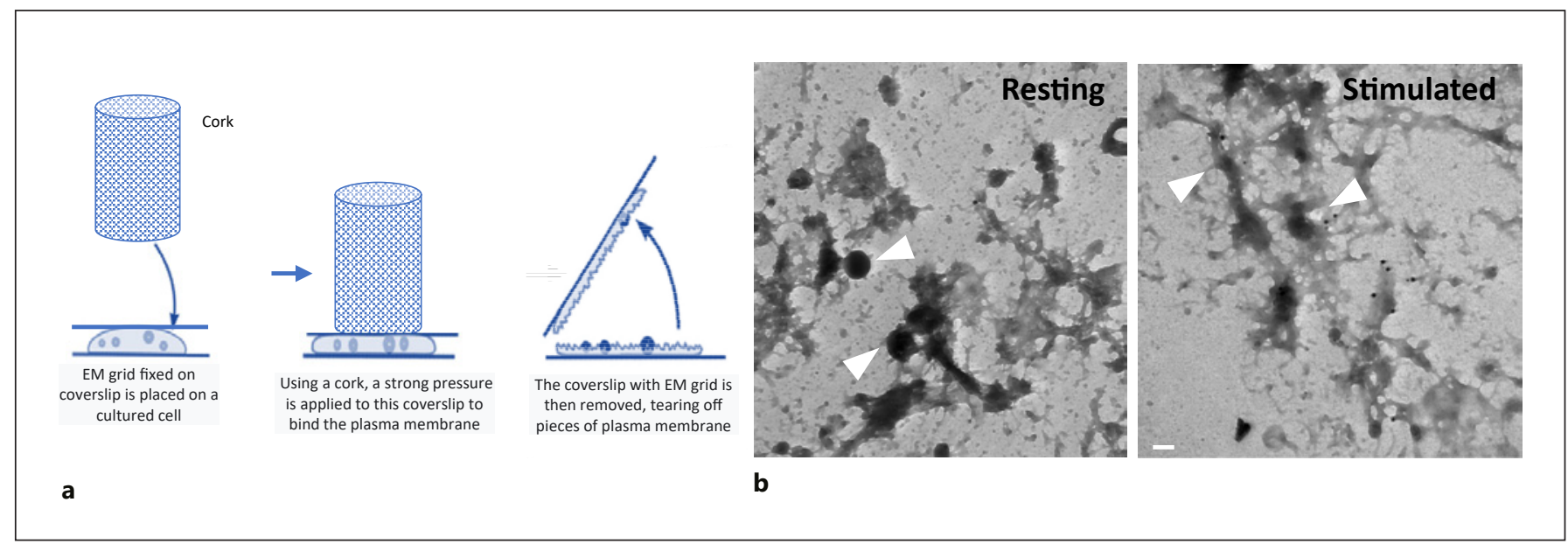

Fig. 2. TEM and secretory granule fusion. TEM operates on the same basic principles as the light microscope but uses electrons instead of photons to pass through the specimen. Because the wavelength of electrons is much smaller than that of light, the optimal resolution attainable for TEM images is many orders of magnitude better than that from a light microscope. Thus, TEM reveals the finest details of cell internal structure - in some cases as small as individual atoms. TEM is frequently used to study organelle morphology. a Preparation of plasma membrane sheets for TEM imaging. b Images of plasma membrane sheets obtained using TEM. Few secretory granules docked at the plasma membrane are observed on plasma membrane sheets of resting cell, while exocytotic sites are observed on plasma membrane sheets of stimulated cells after secretory granule fusion (white arrow heads). Exocytotic sites are identified using an immunogold protocol. Scale bar $100 \mathrm{~nm}$. lar mechanisms involved in neuroendocrine secretion (Fig. 3). The resolution obtained by wide-field microscopy is limited by the Abbe's [46] diffraction limit, defined as the minimum distance between two closely localized structures that can be distinguished from each other. The achieved resolution for understanding cellular and subcellular structures is restrained to $\sim 250 \mathrm{~nm}$ in the lateral plane and $\sim 500-700 \mathrm{~nm}$ in the axial dimension $[47$, 48]. This limit was overcome by fluorescence nanoscopy, also referred to as SRM. In this section, we review the most relevant contributions to the development of SRM for the study of molecular events in neuroendocrine cells. The three main types of SRM used to study neuroendocrine secretion are STED, single-molecule localization microscopy (SMLM), and SIM. These techniques are briefly described in Figure 3.

\section{Stimulated Emission Depletion}

The main advantage of STED is that it allows image acquisition at a resolution below the diffraction limit, achieving a resolution of $\sim 40 \mathrm{~nm}$ laterally and $\sim 150 \mathrm{~nm}$ axially (Fig. 3). STED microscopy has been used in several recent studies to examine the dynamics and implications of fusion pores in living neuroendocrine chromaffin cells. Shin et al. [49] were able to observe the opening, expansion, constriction, and closure dynamics of 0-490 $\mathrm{nm}$ pores and then determine the fusion and fission efficiency during exocytosis and endocytosis events, efficiency being defined by the rate of releasing contents and retrieving vesicles. In another study, Abbineni et al. [50] analyzed the behavior and implications of fused granule membranes using a GFP-tagged pleckstrin homology domain of PLC $\delta 1$. The authors observed the diffusion of the GFP-tagged pleckstrin homology domain of PLC $\delta 1 / \mathrm{Pt}$ dIns(4,5)P2 complex from the cytosolic leaflet of the plasma membrane to the fused secretory granule membrane and were able to determine that diffusion would occur within tens of milliseconds rather than milliseconds [50]. Other studies using STED demonstrated that the dynamic assembly of several proteins (F-actin, N-WASP, and formin) mediates omega-profile merging of the fusion pore by providing sufficient plasma membrane tension to shrink the omega-profile $[39,51]$.

\section{Structured Illumination Microscopy}

The SIM technique is based on the illumination of the sample with a series of excitation light patterns (Fig. 3), which cause a lateral resolution approximately twofold higher than that of confocal microscopy [52]. Compared to other SRM methods, SIM has the particularity to image thicker sections for 3D- and live-cell imaging (Table 1). Moreover, subsecond temporal resolution can be ob- 


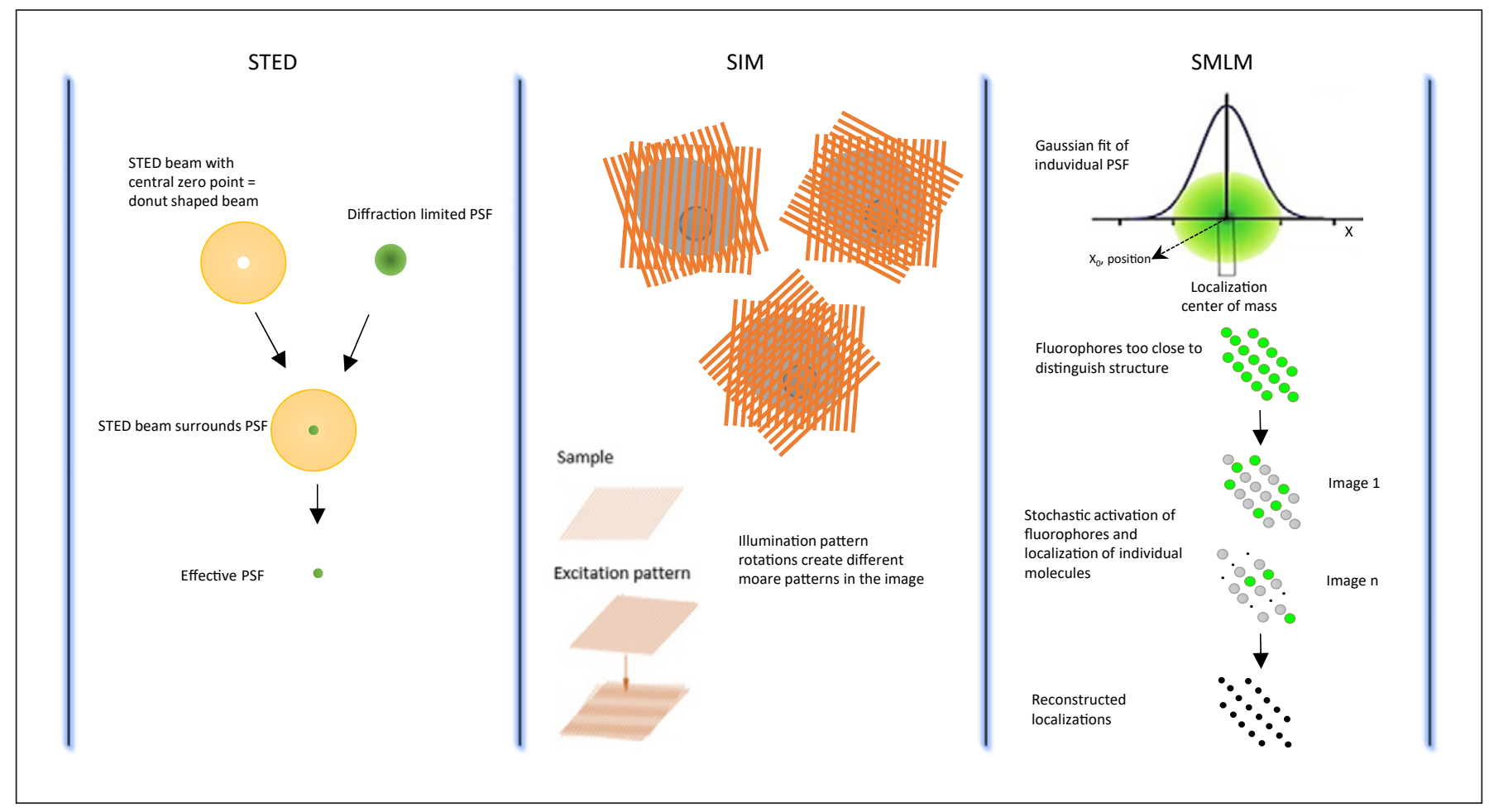

Fig. 3. An overview of super-resolution microscopy techniques. The STED microscopy technique relies on the use of a specific illumination method and photo-physics to generate directly highly resolved images. Super-resolution images are achieved by reducing the size of the effective PSF. This reduction is obtained by illuminating the sample with two aligned beams: a confocal excitation beam to excite fluorescence and a donut-shaped beam. The resulting spot in the middle of the donut, from which the normal emission occurs, can be as small as 40 to $80 \mathrm{~nm}$ large. Basically, only the fluorophores close to the center of the donut will emit, resulting in a reduced PSF and therefore improved resolution. The generation of an image is similar to classical confocal microscopy, but due to the very small voxel size, the scanning speed is relatively slow. Typically, STED can reach a lateral resolution of 150-300 nm with a temporal resolution of seconds, comparable to that of a confocal microscope. SMLM encompasses several related techniques (such as STORM and PALM) that obtain many thousands of images of a given sample in which only a very small number of fluorophores are fluorescing at a given time. Precise localization of the geometric centers of individual fluorophores within the thousands of collected images can then be used to reconstruct a super-resolutive image. The SIM technique relies on the illumination of a sample using high spatial frequency patterns. The excitation light has parallel stripe lines. Then, the combination of the patterned illumination and the sample generates large, detectable interference patterns. The stripe pattern of light, which is applied to the cell, is shifted and rotated so that the entire cell is illuminated within several images. These super-resolution images contain sub-diffraction-limited structural information and can be mathematically deconvolved from the interference signal. PSF, point spread function. tained without high illumination power, making SIM appropriate for use with living cells [53]. In chromaffin cells, SIM has been used to visualize and analyze the spatial correlation between secretory granules and F-actin [54, 55]. SIM has also been used to show that the cellular distribution of secretory granules was not affected by $\mathrm{t}$ SNARE and Munc18-2 overexpression [56]. SIM imaging has allowed the tracking of secretory granule biogenesis, revealing that secretory granules separate from an intermediate Golgi compartment, mature in its proximity for about $1 \mathrm{~h}$, and then travel to the plasma membrane [57]. At a molecular level, 3D-SIM has revealed that the exocy- totic machinery composed of vSNAREs and synaptotagmins is most likely acquired during secretory granule maturation and that recycling of secretory granule membrane proteins is achieved in less than $2 \mathrm{~h}$ [58]. In Tawfik et al.'s [58] study, 3D-SIM, through its high lateral resolution and its ability to distinguish single objects in distinct channels, has also revealed that CgA-containing secretory granules are distinct from synaptotagmin spots.

\section{Single-Molecule Localization Microscopies}

Photoactivated localization microscopy (PALM) and stochastic optical reconstruction microscopy (STORM) 


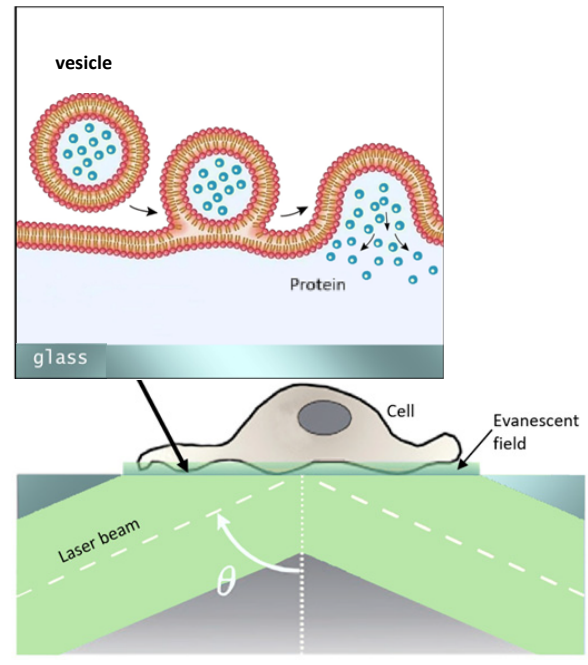

a

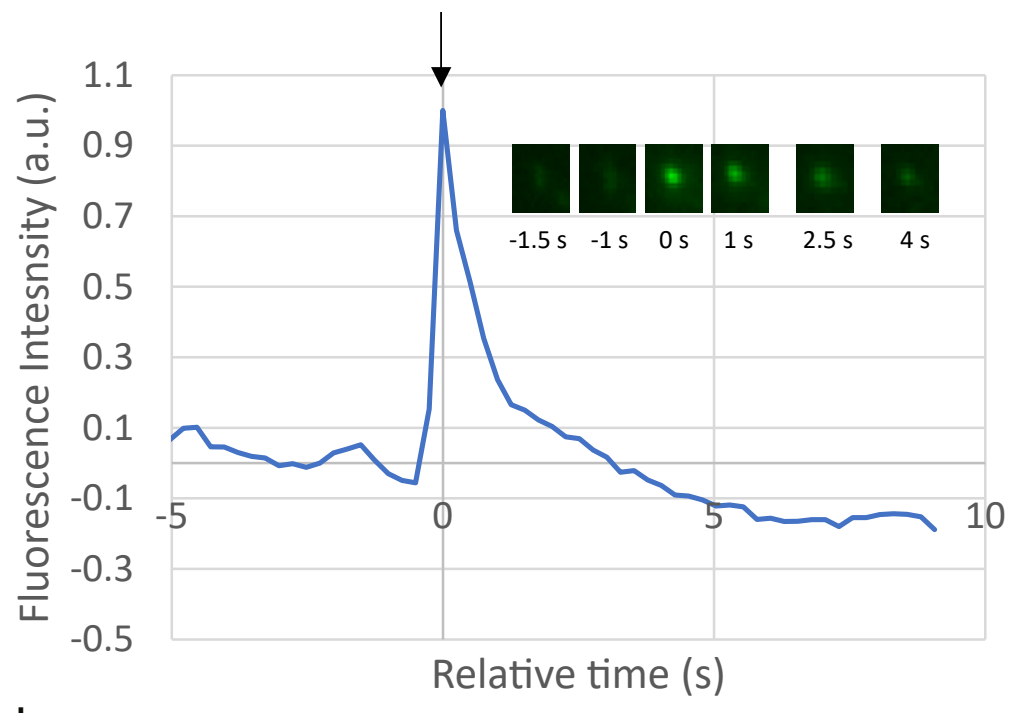

Fig. 4. TIRFM and neuropeptide release kinetics. a TIRFM is an optical technique that provides the excitation of fluorophores in a thin axial region. The method is based on the principle that the optical sectioning of TIRF relies on the excitation confinement of the evanescent wave generated at the glass/cell interface. Since the intensity of the evanescent wave exponentially decays with distance from the surface of the coverslip, only fluorescent molecules within a few hundred nanometers of the surface are effi- ciently excited. In living neuroendocrine cells expressing CgAEGFP, TIRFM was used to track CgA-containing secretory vesicles near to the plasma membrane upon time after secretion stimulation. b Images resulting from a single secretory granule tracking display a fluorescence intensity variation that is plotted and revealed a fast and high increase of fluorescence (arrow) due to the CgA-EGFP release consecutive to secretory granule fusion during exocytosis. are wide-field fluorescence microscopy methods (as opposed to point-scanning techniques such as laser scanning confocal microscopy) that enable single-molecule imaging with a resolution beyond the diffraction limit (Fig. 3). The methods were proposed in 2006 in the wake of a general emergence of optical SRM methods and were featured as methods of the year for 2008 by the $\mathrm{Na}$ ture Methods journal. The high resolution obtained with STORM has revealed three-dimensional ultrastructure of the actin cytoskeleton [59] and nano-sized syntaxin clusters [60] in fixed cells. PALM has been used to study fixed cells but also live cells, and its combination with single-particle tracking allows a high temporal resolution (Table 1) [61]. Then, this SMLM technique enabled to analyze the distribution and mobility of individual synaptic proteins in membrane nanoclusters, such as syntaxin1A [62-64], voltage-gated $\mathrm{Ca}^{2+}$ channels [65, $66]$, postsynaptic AMPA receptors $[67,68]$, and actin [69].

\section{Total Internal Reflection Fluorescence Microscopy}

One of the first fluorescence methods developed to study the real-time dynamics near/of the plasma membrane was TIRF microscopy, also known as evanescent field microscopy [70-72] (Fig. 4). Fluorescence excitation is limited to an optical slice near a glass/water interface. Only a small thickness of the ventral part of the fluorescent dye-loaded cell body is illuminated. To create the evanescent excitation, a high numerical aperture $(>1.4)$ objective is used to allow the total reflection of the laser at the interface and to collect the signal [73]. In this configuration, the greater the laser reflection angle, the thinner the evanescent field. The field of excitation light can be $50 \mathrm{~nm}$ depth, which illuminates the membrane vicinity but leaves the rest of the cell unstimulated/dark, producing a high-contrast, low-background image of the glass-attached plasma membrane [74]. TIRFM is widely used to localize focal adhesion zones and to study local dynamics of structures at the level of the plasma membrane, such as synaptic vesicles, at millisecond time reso- 
lution in living cells $[75,76]$. Evanescent excitation significantly limits the photodamage of the specimen which explains the potential of TIRF microscopy to track dynamics of single secretory granules fusing with the plasma membrane, endocytic vesicles retrieving cargo, and single-receptor dynamics at the nanometer scale in living cells $[70,77]$. To improve the image resolution, it must be noted that TIRFM has recently been applied to isolated plasma membranes coming from live neuroendocrine cells encoding a fluorescent granular protein to study endocytosis and exocytosis events [78, 79].

Many improved versions of TIRF adapted to modern TIRF setups have been developed, such as variable-angle TIRFM with a nanometric axial resolution, typically 10 $20 \mathrm{~nm}$. This setup has been used to measure the axial motion of secretory granules in the basal side of living cells [80]. Another modification of TIRF has been obtained by varying the polarization of the excitation beam to generate a polarized excitation field $[50,81]$. Recently, this method has been used to monitor the real-time shape of exocytotic secretory granules during fusion [50] and the curvature of clathrin-coated pits during endocytosis [82] in chromaffin cells. These studies have provided direct insights into how membranes bend during fusion and fission events, showing the dynamic heterogeneity of these plasma membrane/organelle systems in living cells. Abbineni et al. [83] have shown that the fusion pore curvature of $\mathrm{CgA}$-containing secretory granules is long lived, indicating that fusion pore expansion can be controlled by a lumen protein during regulated exocytosis in chromaffin cells. TIRFM has been coupled to patch-clamp membrane capacitance measurements and carbon-fiber amperometry to demonstrate that, in chromaffin cells, exocytosis could be $\mathrm{Ca}^{2+}$-independent and tightly coupled to membrane depolarization to support secretion during action potentials at low basal rates [84]. A new approach coupling TIRFM with a microelectrode array serves as a powerful platform to record and visualize simultaneously the action potentials and vesicular exocytosis in neuroendocrine PC12 cells [85]. The combination of optical and electrical techniques enables mapping of neuron connectivity, revealing the relevance of electrical and chemical activities in the neuronal model. Other modifications of TIRFM that have gained prominence over the last decade are the result of its merger with superresolution techniques. Harris et al. [86] combined TIRFM and AFM force clamp to identify the nanomechanical properties of the secretory granule-plasma membrane tether by force clamp measurements. They prepared plasma membrane sheets from PC12 cells to locate and track

Innovative Imaging Techniques to Study

Neuroendocrine Secretion the movement of secretory granules attached to the AFM cantilever tip. Their findings showed that the frequency and length of tether unfolding events were consistent with those of exocyst complex helices, indicating the tether extensions play a significant role in the secretory granule-plasma membrane link. A distribution of tether extension steps was observed around $\sim 5 \mathrm{~nm}$. Evanescent field illumination has also been combined with SIM and SMLM in order to reduce an out-of-focus signal [87]. One of these methods, called TIRF-SIM, is able to break the diffraction limit by using patterned illumination that leads to the reconstruction of an image with a resolution twice as good as that of a conventional TIRF microscope without compromising imaging speed $[52,88]$. As a result, objects that have structures below the diffraction limit (e.g., actin filaments and microtubules) can be tracked and studied over time in multiple colors [54, 89]. Combination of SMLM with TIRF described the nanoscale locations and dynamic behaviors of single-ion channels in relation to single vesicles in living cells [89].

\section{How to Choose the Most Suitable Imaging Approach to Study Exocytosis and Endocytosis in Neuroendocrine Cells?}

All the nanoscopy techniques mentioned above have different advantages and limitations (Table 1). It is therefore crucial to choose the ideal technique to answer the desired scientific question. The best spatial resolution is acquired with AFM (typically $1 \mathrm{~nm}$, down to $0.1 \mathrm{~nm}$ ). The major difference between AFM and competing technologies (e.g., light microscopy and TEM) is that AFM does not use lenses or beam irradiation, both of which induce cell damage. Moreover, it is not limited in spatial resolution due to diffraction and aberration, and neither preparing a space for guiding the beam (by creating a vacuum) nor staining the sample are necessary. Therefore, this technique has been used to provide a precise measurement of the 3D morphology of fusion pores after neuroendocrine-cell stimulation. Even if AFM is more often used to study fixed cells, its evolution goes toward an application on living cells [30,31].

TEM offers better resolution for visualizing secretory granule morphology than what can be achieved by current SRM techniques. However, electrons passing through or scattering off the sample interact only weakly with carbon-based matter which explains the unavoidable sample treatment with stains and fixatives [36]. For the aforementioned reasons, fluorescence microscopy has 2 ad- 
vantages: (1) the requirements for sample preparation and observation are modest and make fewer changes to the original form and (2) dynamics events may be observed and studied in living cells. The main limitation of fluorescence techniques is that fluorescent protein overexpression may change organelle behavior, as has been reported for secretory granule fusion during the exocytosis process [83]. Although it is not necessarily true for all advanced, super-resolution, light microscopy realizations, in principle, light microscopy allows observation of structures inside a living cell in real time. Among the types of scanning microscopy techniques including scanning probe microscopy (i.e., AFM, scanning tunneling microscopy, STED, and electrochemical AFM), STED provides intermediate spatial and temporal resolution and fast data acquisition. STED improvement enables better image quality by removing autofluorescence while preserving the desired signal and the separation of fluorescent species even when their emissions fully overlap. Moreover, since STED uses visible, infrared, or even terahertz light to illuminate the sample, image resolution is not constrained by the diffraction limit. The major limitation of this technique is the restricted choice of dyes resistant to the photobleaching induced by STED illumination mode. This characteristic also induces cell photodamage, which led to the use of STED more often on fixed cells. Recently, this super-resolution technique used lifetime imaging which enables to explore the molecular mechanisms involved in multiple dynamic events simultaneously in living cells (e.g., exocytosis and endocytosis in neuroendocrine cells) [90]. A brief overview and practical comparison of commercial implementations of STED, SIM, and SMLM have been published [91]. While the fastest data acquisition is achieved using STED, SIM has fewer requirements regarding sample preparation and gives the best signal-to-noise ratio. Of all commercial implementations, SMLM is likely to provide the best image resolution (typically $20 \mathrm{~nm}$, down to $5 \mathrm{~nm}$ in special cases), with a high temporal resolution in the case of single-particle tracking PALM (Table 1). However, it requires acquisition of thousands raw frames to produce an image and large data processing, which do hamper livecell imaging [92]. SMLM and STED also require the use of special probes (photoswitchable dyes) and experimental conditions. Therefore, SMLM is preferred for static measurements where maximum resolution is needed and when information at the single-molecule level is desired. Since a high density of labeling is necessary to achieve optimal resolution and the dye structure can have a significant effect on molecular properties, these aspects can result in perturbation of the structure of interest. Special care must be taken in the experimental design and sample preparation to prevent this from occurring. The validation of SMLM clustering methods remains a challenge. Potential future directions using multi-modality imaging (e.g., SMLM coupled to electron microscopy) might help validate quantitative SMLM image analysis methods [93]. TIRFM and SIM enable easy and reasonably fast imaging with standard fluorophores. SIM and TIRFM also require less illumination power than SMLM and STED and therefore are suggested for photosensitive samples. The $\mathrm{z}$ resolution of TIRF (typically $50 \mathrm{~nm}$ ) is better than SIM (typically $300 \mathrm{~nm}$ ) and worse than that of SMLM and STED, but the confinement of excitation light to a thin, nearinterface layer in TIRFM configuration results in background reduction and contrast enhancement without being invasive and causing damage.

Because of these characteristics, SIM, TIRFM, and STED methods of cell imaging are commonly used to study various aspects of the neurosecretion process in living neuroendocrine cells (e.g., secretory granule biogenesis, exocytosis, and retrieving, including fusion pore and recycling vesicle dynamics, structure, and single-molecule dynamics) at the nanometer scale. Optimized versions of pioneer resolutive techniques have been developed and are currently used to finely visualize and track single molecules, such as CLEM and HS-AFM. It is interesting to note that, to study the molecular interaction between secretory granule and plasma membranes during exocytosis in living cells, several fluorescence microscopy techniques (TIRFM, SIM, and SMLM) can be implemented with some specialized fluorescence characterization techniques, e.g., Förster resonance energy transfer, fluorescence lifetime imaging, and with fluorescence correlation spectroscopy (module. Among these techniques, the fluorescence lifetime imaging-Förster resonance energy transfer combination can discriminate nanoclusters of synaptic proteins exhibiting variable degrees of interactions with labeled binding partners [94, 95], and fluorescence correlation spectroscopy allows the precise quantification of interaction-induced modifications of diffusion characteristics of membrane molecules at the nanoscale, occurring within short period of times [96].

\section{Future}

High-resolution microscopy, such as SMLM, STED, TIRFM, and SIM, each have distinctive and valuable techniques for the study of neuroendocrine cell structure and 
dynamics, ideally complementing TEM and AFM. Highresolution microscopy alone is not sufficient to adequately study neuroendocrine secretion; therefore, studies still must rely on TEM. CLEM, which combines SRM and EM, has been developed to solve this problem. The purpose of these nanoscale techniques is to visualize the precise localization of single proteins within the machinery driving exocytosis and endocytosis processes, thereby highlighting the molecular mechanism at the origin of secretory granule fusion and retrieving. Current studies are attempting to obtain $3 \mathrm{D}$ resolution in multicolor toward the nanometer scale and high-speed imaging to determine the molecular mechanisms governing neuroendocrine secretion at the cellular level. Recently, another point-scanning fluorescent method with a high $3 \mathrm{D}$ imaging performance, called minimal emission fluxes nanoscopy, has been developed to break the diffraction limit while imaging dynamic single events in living cells [97]. This technique requires fewer detected photons than standard camera-based localization and allows the use of a larger range of fluorophores. Consequently, minimal emission fluxes enables nanometer scale tracking of molecules with temporal resolution less than $1 \mathrm{~ms}$ [98]. While these technological developments have made significant contributions, it is essential to develop reference standards for quality control, counting of protein copy numbers in complexes, and to develop software to extract specific and quantitative information from SRM data for biological interpretation. Furthermore, fluorescent techniques are often applied in studying overexpressed fusion proteins that can help obtain the labeling required for high resolution but can result in aberrant protein expressions with functional consequences [99]. To overcome the biological disruption, recent studies reported advances in the field using the CRISPR/Cas9 approach toward studying endogenously tagged proteins for single-molecule imaging techniques $[100,101]$. The challenges of all these advents will benefit to the neuroendocrinologist community in the sense that they aim to provide more precise visualization, tracking, and quantification of molecule association in situ in living neuroendocrine cells, with the least damages and/or modifications, facilitating our understanding of molecular interactions that drive neurosecretion in any physiological context.

\section{Conflict of Interest Statement}

The authors declare that no competing interests exist.

\section{Funding Sources}

This work was supported by institutional funding from INSERM, University of Rouen-Normandie, IBiSA, IRIB, Région Normandie, the European Regional Development Funds (RIN Recherche 2019 Emergents PA-TOOLBOX) to MM-H and grant from the Agence Nationale de la Recherche (ANR) (ANR PA-Box number ANR-19-CE44-0019) to MM-H. L.R. and T.F. were supported by fellowships from ANR.

\section{Author Contributions}

L. Riachy wrote the manuscript with significant inputs from $\mathrm{T}$. Ferrand (TIRFM section), S. Chasserot-Golaz (TEM section), L. Galas (super-resolution section), and S. Alexandre (AFM section); M. Montero-Hadjadje coordinated the writing of the paper.

\section{References}

1 Huttner WB, Tooze SA. Biosynthetic protein transport in the secretory pathway. Curr Opin Cell Biol. 1989;1(4):648-54.

2 Tooze SA. Biogenesis of secretory granules in the trans-Golgi network of neuroendocrine and endocrine cells. Biochim Biophys Acta. 1998;1404(1-2):231-44.

3 Steyer JA, Horstmann H, Almers W. Transport, docking and exocytosis of single secretory granules in live chromaffin cells. Nature. 1997;388(6641):474-8.

4 Meunier FA, Gutiérrez LM. Captivating new roles of F-actin cortex in exocytosis and bulk endocytosis in neurosecretory cells. Trends Neurosci. 2016;39(9):605-13.

5 Holz RW, Axelrod D. Secretory granule behaviour adjacent to the plasma membrane be- fore and during exocytosis: total internal reflection fluorescence microscopy studies. Acta Physiol. 2008;192(2):303-7.

6 Burgoyne RD, Morgan A. Regulated exocytosis. Biochem J. 1993;293(Pt 2):305-16.

7 Gondré-Lewis MC, Park JJ, Loh YP. Cellular mechanisms for the biogenesis and transport of synaptic and dense-core vesicles. Int Rev Cell Mol Biol. 2012;299:27-115.

8 Laguerre F, Anouar Y, Montero-Hadjadje M. Chromogranin A in the early steps of the neurosecretory pathway. IUBMB Life. 2020 Apr; 72(4):524-32.

9 Yang R, Xi N, Fung CK, Seiffert-Sinha K, Lai KW, Sinha AA. The emergence of AFM applications to cell biology: how new technologies are facilitating investigation of human cells in health and disease at the nanoscale. J Nanosci Lett. 2011;1 (2):87-101. PMID: 24416719.

10 Tanguy E, Thahouly T, Royer C, Demais V, Gasman S, Chasserot-Golaz S, et al. Protocol for electron microscopy ultrastructural localization of the fusogenic lipid phosphatidic acid on plasma membrane sheets from chromaffin cells. STAR Protoc. 2021;2(2): 100464. http://dx.doi.org/10.1016/j.xpro.2021.100464.

11 Vangindertael J, Camacho R, Sempels W, Mizuno H, Dedecker P, Janssen KPF. An introduction to optical super-resolution microscopy for the adventurous biologist. Methods Appl Fluoresc. 2018;6(2):22003.

12 Binning G, Rohrer H, Gerber C, Weibel E. Surface studies by scanning tunneling microscopy. Phys Rev. 1982;49(1):6196. 
13 Zhang H, Huang J, Wang Y, Liu R, Huai X, Jiang J, et al. Atomic force microscopy for two-dimensional materials: a tutorial review. Opt Commun. 2018;406:3-17.

14 Zhang J, Zeng H. Intermolecular and surface interactions in engineering processes. Engineering. 2021;7(1):63-83.

15 Kubota R, Tanaka W, Hamachi I. Microscopic imaging techniques for molecular assemblies: electron, atomic force, and confocal microscopies. Chem Rev. 2021;121(22).

16 Liang W, Shi H, Yang X, Wang J, Yang W, Zhang $\mathrm{H}$, et al. Recent advances in AFMbased biological characterization and applications at multiple levels. Soft Matter. 2020; 16(39):8962-84.

17 Amarouch MY, El Hilaly J, Mazouzi D. AFM and FluidFM technologies: recent applications in molecular and cellular biology. Scanning. 2018;2018:1-10.

18 Stylianou A, Kontomaris S-V, Grant C, Alexandratou $\mathrm{E}$. Atomic force microscopy on biological materials related to pathological conditions. Scanning. 2019;2019.

19 Chang K-C, Chiang Y-W, Yang C-H, Liou $\mathrm{J}-\mathrm{W}$. Atomic force microscopy in biology and biomedicine. Tzu Chi Med J. 2012;24(4):1629.

20 Tsai CC, Lin CL, Wang TL, Chou AC, Chou $\mathrm{MY}$, Lee $\mathrm{CH}$, et al. Dynasore inhibits rapid endocytosis in bovine chromaffin cells. Am J Physiol Cell Physiol. 2009 Aug;297(2):C397406.

21 Geisse NA. AFM and combined optical techniques. Mater Today. 2009;12(7-8):40-5.

22 Dufrêne YF. Atomic force microscopy in microbiology: new structural and functional insights into the microbial cell surface. mBio. 2014;5(4):1-14.

23 Hamon L, Curmi PA, Pastré D. High-resolution imaging of microtubules and cytoskeleton structures by atomic force microscopy. Methods Cell Biol. 2010;95:157-74.

24 Deng X, Xiong F, Li X, Xiang B, Li Z, Wu X, et al. Application of atomic force microscopy in cancer research. J Nanobiotechnology. 2018;16(1)

25 Lekka M, Pabijan J. Measuring elastic properties of single cancer cells by AFM. Methods Mol Biol. 2019;1886:315-24.

26 Tsai CC, Yang CC, Shin PY, Wu CS, Chen $\mathrm{CD}$, Pan CY, et al. Exocytosis of a single bovine adrenal chromaffin cell: the electrical and morphological studies. J Phys Chem B. 2008 Jul;112(30):9165-73.

27 Robertson AS, Smythe E, Ayscough KR. Functions of actin in endocytosis. Cell $\mathrm{Mol}$ Life Sci. 2009;66(13):2049-65.

28 Carmon O, Laguerre F, Riachy L, DelestreDelacour C, Wang Q, Tanguy E, et al. Chromogranin A preferential interaction with Golgi phosphatidic acid induces membrane deformation and contributes to secretory granule biogenesis. FASEB J. 2020;34(5): 6769-90.

29 Tsai CC, Hung HH, Liu CP, Chen YT, Pan $\mathrm{CY}$. Changes in plasma membrane surface potential of PC12 cells as measured by Kelvin probe force microscopy. PLoS One. $2012 \mathrm{Apr}$; 7(4):e33849.

30 Dulebo A, Preiner J, Kienberger F, Kada G, Rankl C, Chtcheglova L, et al. Second harmonic atomic force microscopy imaging of live and fixed mammalian cells. Ultramicroscopy. 2009;109(8):1056-60.

31 Cartagena-Rivera AX, Wang WH, Geahlen RL, Raman A. Fast, multi-frequency, and quantitative nanomechanical mapping of live cells using the atomic force microscope. Sci Rep. 2015;5(1):11692.

32 Schillers H, Medalsy I, Hu S, Slade AL, Shaw JE. PeakForce tapping resolves individual microvilli on living cells. J Mol Recognit. 2016; 29(2):95-101.

33 Zhang X, Ren J, Wang J, Li S, Zou Q, Gao N. Receptor-mediated endocytosis generates nanomechanical force reflective of ligand identity and cellular property. J Cell Physiol. 2018;233(8):5908-19.

34 Casuso I, Redondo-Morata L, Rico F. Biological physics by high-speed atomic force microscopy. Philos Trans A Math Phys Eng Sci. 2020;378(2186):20190604.

35 Shibata M, Watanabe H, Uchihashi T, Ando T, Yasuda R. High-speed atomic force microscopy imaging of live mammalian cells. Biophys Physicobiol. 2017;14:127-35.

36 Koval LM, Yavorskaya EN, Lukyanetz EA. Electron microscopic evidence for multiple types of secretory vesicles in bovine chromaffin cells. Gen Comp Endocrinol. 2001;121(3): 261-77.

37 Heuser JE, Reese TS. Structural changes after transmitter release at the frog neuromuscular junction. J Cell Biol. 1981;88(3):564-80.

38 Zhao WD, Hamid E, Shin W, Wen PJ, Krystofiak ES, Villarreal SA, et al. Hemi-fused structure mediates and controls fusion and fission in live cells. Nature. 2016;534(7608):548-52.

39 Wen PJ, Grenklo S, Arpino G, Tan X, Liao HS, Heureaux J, et al. Actin dynamics provides membrane tension to merge fusing vesicles into the plasma membrane. Nat Commun. 2016 Aug; 7:12604.

40 Gabel M, Delavoie F, Demais V, Royer C, Bailly Y, Vitale N, et al. Annexin A2-dependent actin bundling promotes secretory granule docking to the plasma membrane and exocytosis. J Cell Biol. 2015;210(5):785-800.

41 Tanguy E, Wolf A, Montero-Hadjadje M, Gasman S, Bader MF, Vitale N. Phosphatidic acid: mono- and poly-unsaturated forms regulate distinct stages of neuroendocrine exocytosis. Adv Biol Regul. 2020;79:100772.

42 Sjollema KA, Schnell U, Kuipers J, Kalicharan $\mathrm{R}$, Giepmans BNG. Correlated light microscopy and electron microscopy. Methods Cell Biol. 2012;111:157-73.

43 De Boer P, Hoogenboom JP, Giepmans BNG. Correlated light and electron microscopy: ultrastructure lights up. Nat Methods. 2015; 12(6):503-13

44 Sochacki KA, Shtengel G, Van Engelenburg SB, Hess HF, Taraska JW. Correlative super- resolution fluorescence and metal-replica transmission electron microscopy. Nat Methods. 2014;11(3):305-8.

45 Prasai B, Haber GJ, Strub MP, Ahn R, Ciemniecki JA, Sochacki KA, et al. The nanoscale molecular morphology of docked exocytic dense-core vesicles in neuroendocrine cells. Nat Commun. 2021;12(1):1-14.

46 Abbe E. Beiträge zur theorie des mikroskops und der mikroskopischen wahrnehmung. Arch für Mikroskopische Anat. 1873;9(1):418-40.

47 Dodgson J, Chessel A, Cox S, Carazo Salas RE. Super-resolution microscopy: SIM, STED and localization microscopy. In: Dahms TES, Czymmek KJ, editors. Advanced microscopy in mycology. Springer; 2015. p. 47-60.

48 Vangindertael J, Camacho R, Sempels W, Mizuno H, Dedecker P, Janssen KPF. An introduction to optical super-resolution microscopy for the adventurous biologist. Methods Appl Fluoresc. 2018;6(2):22003.

49 Shin W, Ge L, Arpino G, Villarreal SA, Hamid $\mathrm{E}$, Liu $\mathrm{H}$, et al. Visualization of membrane pore in live cells reveals a dynamic-pore theory governing fusion and endocytosis. Cell. 2018 May;173(4):934-45.e12.

50 Abbineni PS, Axelrod D, Holz RW. Visualization of expanding fusion pores in secretory cells. J Gen Physiol. 2018 Dec;150(12):1640-6.

51 Bottanelli F, Kromann EB, Allgeyer ES, Erdmann RS, Baguley SW, Sirinakis G, et al. Twocolour live-cell nanoscale imaging of intracellular targets. Nat Commun. 2016;7:10778.

52 Gustafsson MG. Surpassing the lateral resolution limit by a factor of two using structured illumination microscopy. J Microsc. 2000; 198(2):82-7.

53 Godin AG, Lounis B, Cognet L. Super-resolution microscopy approaches for live cell imaging. Biophys J. 2014;107(8):1777-84.

54 Bost A, Pasche M, Schirra C, Becherer U. Super-resolution microscopy in studying neuroendocrine cell function. Front Neurosci. 2013;7:222.

55 Kurps J, Broeke JH, Cijsouw T, Kompatscher A, van Weering JR, de Wit H. Quantitative image analysis tool to study the plasma membrane localization of proteins and cortical actin in neuroendocrine cells. J Neurosci Methods. 2014 Oct;236:1-10.

56 Hugo S, Dembla E, Halimani M, Matti U, Rettig J, Becherer U. Deciphering dead-end docking of large dense core vesicles in bovine chromaffin cells. J Neurosci. 2013;33(43): 17123-37.

57 Dembla E, Becherer U. Biogenesis of large dense core vesicles in mouse chromaffin cells. Traffic. 2021;22(3):78-93.

58 Tawfik B, Martins JS, Houy S, Imig C, Pinheiro PS, Wojcik SM, et al. Synaptotagmin-7 places dense-core vesicles at the cell membrane to promote munc13-2-and ca2+-dependent priming. Elife. 2021;10:1-40.

59 Xu K, Babcock HP, Zhuang X. Dual-objective STORM reveals three-dimensional filament organization in the actin cytoskeleton. Nat Methods. 2012;9(2):185-8. 
60 Bar-On D, Wolter S, Van De Linde S, Heilemann M, Nudelman G, Nachliel E, et al. Super-resolution imaging reveals the internal architecture of nano-sized syntaxin clusters. J Biol Chem. 2012;287(32):27158-67.

61 Manley S, Gillette JM, Patterson GH, Shroff $\mathrm{H}$, Hess HF, Betzig E, et al. High-density mapping of single-molecule trajectories with photoactivated localization microscopy. Nat Methods. 2008;5(2):155-7.

62 Bademosi AT, Lauwers E, Padmanabhan P, Odierna L, Chai YJ, Papadopulos A, et al. In vivo single-molecule imaging of syntaxin $1 \mathrm{~A}$ reveals polyphosphoinositide- and activitydependent trapping in presynaptic nanoclusters. Nat Commun. 2017;8:13660.

63 Padmanabhan P, Bademosi AT, Kasula R, Lauwers E, Verstreken P, Meunier FA. Need for speed: super-resolving the dynamic nanoclustering of syntaxin-1 at exocytic fusion sites. Neuropharmacology. 2020;169:107554.

64 Bademosi AT, Steeves J, Karunanithi S, Zalucki OH, Gormal RS, Liu S, et al. Trapping of syntaxinla in presynaptic nanoclusters by a clinically relevant general anesthetic. Cell Rep. 2018;22(2):427-40.

65 Schneider R, Hosy E, Kohl J, Klueva J, Choquet $\mathrm{D}$, Thomas $\mathrm{U}$, et al. Mobility of calcium channels in the presynaptic membrane. Neuron. 2015;86(3):672-9.

66 Heck J, Parutto P, Ciuraszkiewicz A, Bikbaev A, Freund R, Mitlöhner J, et al. Transient confinement of CaV2.1 Ca2+-channel splice variants shapes synaptic short-term plasticity. Neuron. 2019;103(1):66-79.e12.

67 Hoze N, Nair D, Hosy E, Sieben C, Manley S, Herrmann A, et al. Heterogeneity of AMPA receptor trafficking and molecular interactions revealed by superresolution analysis of live cell imaging. Proc Natl Acad Sci U S A. 2012;109(42):17052-7.

68 Nair D, Hosy E, Petersen JD, Constals A, Giannone G, Choquet D, et al. Super-resolution imaging reveals that AMPA receptors inside synapses are dynamically organized in nanodomains regulated by PSD95. J Neurosci. 2013:33(32):13204-24.

69 Li P, Bademosi AT, Luo J, Meunier FA. Actin remodeling in regulated exocytosis: toward a mesoscopic view. Trends Cell Biol. 2018; 28(9):685-97.

70 Steyer JA, Almers W. A real-time view of life within $100 \mathrm{~nm}$ of the plasma membrane. Nat Rev Mol Cell Biol. 2001;2(4):268-75.

71 Axelrod R, Hamilton WD. The evolution of cooperation. Science. 1981;211(4489): 1390.

72 Axelrod D. Total internal reflection fluorescence microscopy. Encycl Cell Biol. 2016; 2(2):62-9.

73 Boulanger J, Gueudry C, Münch D, Cinquin B, Paul-Gilloteaux P, Bardin S, et al. Fast highresolution $3 \mathrm{~d}$ total internal reflection fluorescence microscopy by incidence angle scanning and azimuthal averaging. Proc Natl Acad Sci U S A. 2014;111(48):17164-9.
74 Axelrod D. Evanescent excitation and emission in fluorescence microscopy. Biophys J. 2013;104(7):1401-9.

75 Midorikawa M. Real-time imaging of synaptic vesicle exocytosis by total internal reflection fluorescence (TIRF) microscopy. Neurosci Res. 2018;136:1-5.

76 Miki T, Midorikawa M, Sakaba T, Sakaba T. Direct imaging of rapid tethering of synaptic vesicles accompanying exocytosis at a fast central synapse. Proc Natl Acad Sci U S A. 2020; 117(25):14493-502.

77 Mattheyses AL, Simon SM, Rappoport JZ. Imaging with total internal reflection fluorescence microscopy for the cell biologist. J Cell Sci. 2010 Nov; 123(21):3621-8.

78 Milosevic I. Spatial and temporal aspects of phosphoinositides in endocytosis studied in the isolated plasma membranes. Methods Mol Biol. 2018;1847:147-60.

79 Milosevic I. Spatial and temporal aspects of exocytosis studied on the isolated plasma membranes. Methods Mol Biol. 2021;2233:311-25.

80 Loerke D, Stühmer W, Oheim M. Quantifying axial secretory-granule motion with variableangle evanescent-field excitation. J Neurosci Methods. 2002;119(1):65-73.

81 Sund SE, Swanson JA, Axelrod D. Cell membrane orientation visualized by polarized total internal reflection fluorescence. Biophys J. 1999;77(4):2266-83.

82 Scott BL, Sochacki KA, Low-Nam ST, Bailey EM, Luu QA, Hor A, et al. Membrane bending occurs at all stages of clathrincoat assembly and defines endocytic dynamics. Nat Commun. 2018;9(1):419.

83 Abbineni PS, Bittner MA, Axelrod D, Holz RW. Chromogranin A, the major lumenal protein in chromaffin granules, controls fusion pore expansion. J Gen Physiol. $2018 \mathrm{Feb}$; 151(2):118-30.

84 Moya-Díaz J, Bayonés L, Montenegro M, Cárdenas AM, Koch H, Doi A, et al. Ca2+independent and voltage-dependent exocytosis in mouse chromaffin cells. Acta Physiol. 2020 Apr;228(4):e13417.

85 Cui MR, Zhao W, Li XL, Xu CH, Xu JJ, Chen HY. Simultaneous monitoring of action potentials and neurotransmitter release from neuron-like PC12 cells. Anal Chim Acta. 2020 Apr;1105:74-81.

86 Harris MC, Cislo D, Lenz JS, Umbach C, Lindau M. AFM/TIRF force clamp measurements of neurosecretory vesicle tethers reveal characteristic unfolding steps. PLoS One. 2017 Mar;12(3):e0173993.

87 Toomre D, Bewersdorf J. A new wave of cellular imaging. Annu Rev Cell Dev Biol. 2010; $26: 285-314$

88 Guo M, Chandris P, Giannini JP, Trexler AJ, Fischer R, Chen J, et al. Single-shot super-resolution total internal reflection fluorescence microscopy. Nat Methods. 2018;15(6):425-8.
89 Hiersemenzel K, Brown ER, Duncan RR. Imaging large cohorts of single ion channels and their activity. Front Endocrinol. 2013;4 114.

90 Alvarez LAJ, Schwarz U, Friedrich L, Foelling J, Hecht F, Roberti MJ. TauSTED: pushing STED beyond its limits with lifetime. Nat Methods. 2021;1:1-3.

91 Wegel E, Göhler A, Lagerholm BC, Wainman A, Uphoff S, Kaufmann R, et al. Imaging cellular structures in super-resolution with SIM, STED and localisation microscopy: a practical comparison. Sci Rep. 2016;6: 27290.

92 Schermelleh L, Ferrand A, Huser T, Eggeling C, Sauer M, Biehlmaier O, et al. Super-resolution microscopy demystified. Nat Cell Biol. 2019;21(1):72-84.

93 Khater IM, Nabi IR, Hamarneh G. A review of super-resolution single-molecule localization microscopy cluster analysis and quantification methods. Patterns. 2020;1(3): 100038 .

94 Günther E, Klauß A, Toro-Nahuelpan M, Schüler D, Hille C, Faivre D. The in vivo mechanics of the magnetotactic backbone as revealed by correlative FLIM-FRET and STED microscopy. Sci Rep. 2019;9(1):1-9.

95 Tardif C, Nadeau G, Labrecque S, Côté D, Lavoie-Cardinal F, De Koninck P. Fluorescence lifetime imaging nanoscopy for measuring Förster resonance energy transfer in cellular nanodomains. Neurophotonics. 2019;6(1):15002.

96 Wang R, Brustlein S, Mailfert S, Fabre R, Fallet M, Sivankutty S, et al. A straightforward STED-background corrected fitting model for unbiased STED-FCS analyses. Methods. 2018 May;140-1:212-22.

97 Gwosch KC, Pape JK, Balzarotti F, Hoess P, Ellenberg J, Ries J, et al. MINFLUX nanoscopy delivers 3D multicolor nanometer resolution in cells. Nat Methods. 2020;17(2): 217-24.

98 Eilers Y, Ta H, Gwosch KC, Balzarotti F, Hell SW. MINFLUX monitors rapid molecular jumps with superior spatiotemporal resolution. Proc Natl Acad Sci U S A. 2018;115(24): 6117-22.

99 Keren L, Hausser J, Lotan-Pompan M, Vainberg Slutskin I, Alisar H, Kaminski S, et al. Massively parallel interrogation of the effects of gene expression levels on fitness. Cell. 2016;166(5):1282-94.e18.

100 Khan AO, Simms VA, Pike JA, Thomas SG, Morgan NV. CRISPR-Cas9 mediated labelling allows for single molecule imaging and resolution. Sci Rep. 2017;7(1):8450-9.

101 Butkevich AN, Ta H, Ratz M, Stoldt S, Jakobs S, Belov VN, et al. Two-color $810 \mathrm{~nm}$ STED nanoscopy of living cells with endogenous SNAP-tagged fusion proteins. ACS Chem Biol. 2018;13(2):475-80. 